\title{
On the Smallest Point on a Diagonal Cubic Surface
}

\author{
Andreas-Stephan Elsenhans and Jörg Jahnel
}

\section{CONTENTS}

1. Introduction

2. Peyre's Constant

3. A Technical Lemma

4. Splitting the Picard Group

5. The Computation of the $L$-function at 1

6. Computing the Tamagawa Numbers

7. Searching for the Smallest Solution

8. A Negative Result

References
2000 AMS Subject Classification: 11G35, 11G50, 11G40, 14J20, $14 \mathrm{~J} 26$

Keywords: Diagonal cubic surface, Diophantine equation, smallest solution, naive height, E. Peyre's Tamagawa-type number
For diagonal cubic surfaces $S$, we study the behavior of the height $\mathrm{m}(S)$ of the smallest rational point versus the Tamagawatype number $\tau(S)$ introduced by E. Peyre. We determined both quantities for a sample of 849,781 diagonal cubic surfaces. Our methods are explained in some detail. The results suggest an inequality of the type $\mathrm{m}(S)<C(\varepsilon) / \tau(S)^{1+\varepsilon}$. We conclude the article with the construction of a sequence of diagonal cubic surfaces showing that the inequality $\mathrm{m}(S)<C / \tau(S)$ is false in general.

\section{INTRODUCTION}

Let $S \subseteq \mathbf{P}_{\mathbb{Q}}^{n}$ be a Fano variety defined over $\mathbb{Q}$. If $S\left(\mathbb{Q}_{\nu}\right) \neq \varnothing$ for every $\nu \in \operatorname{Val}(\mathbb{Q})$, then it is natural to ask whether $S(\mathbb{Q}) \neq \varnothing$. When this is the case, $S$ is said to satisfy the Hasse principle. Further, it would be desirable to have an a priori upper bound for the height of the smallest $\mathbb{Q}$-rational point on $S$, since this would make it possible to effectively decide whether $S(\mathbb{Q}) \neq \varnothing$.

When $S$ is a conic, Legendre's theorem on zeros of ternary quadratic forms proves the Hasse principle and, moreover, yields an effective bound for the smallest point. For quadrics of arbitrary dimension, the same is true by an observation of [Cassels 55]. Furthermore, [Siegel 69, Satz 1] provides a generalization to hypersurfaces defined by norm equations.

For more general Fano varieties, no theoretical upper bound is known for the smallest height of a $\mathbb{Q}$-rational point. This already applies to diagonal cubic surfaces. Furthermore, some of these fail to satisfy the Hasse principle [Colliot-Thélène et al. 87].

In this article, we present some theoretical and experimental results concerning the smallest height of a $\mathbb{Q}$ rational point on diagonal cubic surfaces in $\mathbf{P}_{\mathbb{Q}}^{3}$.

A conjecture due to Manin asserts that the number of $\mathbb{Q}$-rational points of anticanonical height less than $B$ on a Fano variety $S$ is asymptotically equal to $\tau B \log ^{\text {rk } \operatorname{Pic}(S)-1} B$, for $B \rightarrow \infty$. 
In the particular case of a cubic surface, the anticanonical height is the same as the naive height. Further, the coefficient $\tau \in \mathbb{R}_{\geq 0}$ equals the Tamagawa-type number $\tau(S)$ introduced in [Peyre 95]. Hence, at least $\sim \tau(S) B$ points of height less than $B$ are supposed to exist. Assume that their heights are equally distributed within $[0, B)$. Then the height of the smallest point is less than $1 / \tau(S)$.

Therefore, one might generally expect that $\mathrm{m}(S)$, the height of the smallest $\mathbb{Q}$-rational point on $S$, is bounded by $C / \tau(S)$ for a certain absolute constant $C$.

To test this expectation, we computed the Tamagawa number and ascertained the smallest $\mathbb{Q}$-rational point for each of the cubic surfaces given by

$$
a x^{3}+b y^{3}+2 z^{3}+w^{3}=0
$$

for $a=1, \ldots, 3000$ and $b=1, \ldots, 300$.

We restricted our considerations to the case that

(i) $a$ and $b$ are odd,

(ii) there exists an odd prime $p$ dividing $a$ but not $b$ such that $3 \nmid \nu_{p}(a)$, or

(iii) there exists an odd prime $p$ dividing $b$ but not $a$ such that $3 \nmid \nu_{p}(b)$.

This guarantees that we are in the "first case" according to the classification in [Colliot-Thélène et al. 87].

In addition, we assume that $a>b+3$. The inequality $a \geq b$ is necessary in order to avoid duplications. Further, surfaces such that $|a-b| \leq 3$ trivially have rational points of uncharacteristically small height. The results are summarized in Figure 1. The sample described consists of 849,781 surfaces. Among them, 802,891 turn out to have rational points. Each such surface is marked at its proper place $(\tau(S), \mathrm{m}(S))$ in the diagram.

It is apparent that the experiment agrees with expectation. The slope of a line tangent to the top right of the scatter plot is indeed near $(-1)$. However, as we will show in Theorem 8.1, the inequality $\mathrm{m}(S)<\frac{C}{\tau(S)}$ does not hold in general. The following question remains open as a logical possibility.

Question 1.1. For every $\varepsilon>0$, does there exist a constant $C(\varepsilon)$ such that

$$
\mathrm{m}(S)<\frac{C(\varepsilon)}{\tau(S)^{1+\varepsilon}}
$$

for every cubic surface?

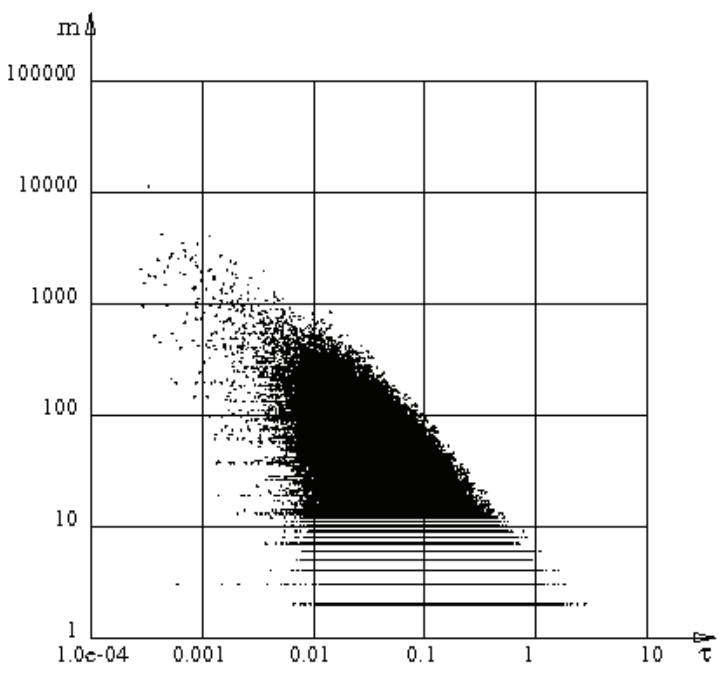

FIGURE 1. Height of smallest point versus Tamagawa number.

\subsection{Plan of the Article}

Sections 2 through 7 will be devoted to the computations that led to the diagram shown as Figure 1.

In Section 2, we will recall Peyre's constant $\tau(S)$. The next four sections will discuss the factors that constitute this constant. First, in Section 3, we will use the Lefschetz trace formula in order to estimate the product over all local factors $\tau_{p}(S)$ at the good primes $p$, uniformly over all cubic surfaces.

Sections 4 and 5 will be concerned with the $L$-factors. In Section 4, for diagonal cubic surfaces, we will give an explicit decomposition of the Galois representation $\operatorname{Pic}(S) \otimes_{\mathbb{Z}} \mathbb{C}$ into irreducible components. As an application of this, we efficiently computed the values of the corresponding Artin $L$-functions at 1 .

Our method will be presented in detail in Section 5. In Section 6, we will explain our approach to dealing with the factor $\tau_{\infty}(S)$. This requires numerical integration. Further, we will describe our computations of the Euler products over all non-Archimedean primes. Finally, in Section 7, we will describe our method for finding the smallest point on every surface in the sample.

Section 8 will be more theoretical in nature. We will construct a sequence $\left\{S^{(q)}\right\}_{q \in \mathbb{N}}$ of diagonal cubic surfaces such that $\mathrm{m}\left(S^{(q)}\right) \tau\left(S^{(q)}\right)$ is unbounded.

Notation 1.2. Let $\mathfrak{a}=\left(a_{0}, \ldots, a_{3}\right) \in(\mathbb{Z} \backslash\{0\})^{4}$ be a vector. Then we denote by $S^{\mathfrak{a}}$ the cubic surface in $\mathbf{P}_{\mathbb{Q}}^{3}$ given by $a_{0} x_{0}^{3}+\cdots+a_{3} x_{3}^{3}=0$. 


\section{PEYRE'S CONSTANT}

Recall that E. Peyre's Tamagawa-type number is defined in [Peyre and Tschinkel 01, Definition 2.4] as

$\tau(S):=\alpha(S) \beta(S) \lim _{s \rightarrow 1}(s-1)^{t} L\left(s, \chi_{\operatorname{Pic}\left(S_{\overline{\mathbb{Q}}}\right)}\right) \tau_{H}\left(S\left(\AA_{\mathbb{Q}}\right)^{\mathrm{Br}}\right)$

for $t=\operatorname{rk} \operatorname{Pic}(S)$. Here, $\operatorname{Pic}(S)$ denotes the Picard group of the $\mathbb{Q}$-scheme $S$.

The factor $\beta(S)$ is simply defined as

$$
\beta(S):=\# H^{1}\left(\operatorname{Gal}(\overline{\mathbb{Q}} / \mathbb{Q}), \operatorname{Pic}\left(S_{\overline{\mathbb{Q}}}\right)\right) .
$$

Further, $\alpha(S)$ is given as follows [Peyre 95, Définition 2.4]. Let $\Lambda_{\text {eff }}(S) \subset \operatorname{Pic}(S) \otimes_{\mathbb{Z}} \mathbb{R}$ be the cone generated by the effective divisors. Identify $\operatorname{Pic}(S) \otimes_{\mathbb{Z}} \mathbb{R}$ with $\mathbb{R}^{t}$ via a mapping induced by an isomorphism $\operatorname{Pic}(S) \stackrel{\cong}{\longrightarrow} \mathbb{Z}^{t}$. Consider the dual cone $\Lambda_{\text {eff }}^{\vee}(S) \subset\left(\mathbb{R}^{t}\right)^{\vee}$. Then

$$
\alpha(S):=t \cdot \operatorname{vol}\left\{x \in \Lambda_{\mathrm{eff}}^{\vee}(S) \mid\langle x,-K\rangle \leq 1\right\} .
$$

Since $L\left(\cdot, \chi_{\operatorname{Pic}\left(S_{\overline{\mathbb{Q}}}\right)}\right)$ denotes the Artin $L$-function of the $\operatorname{Gal}(\overline{\mathbb{Q}} / \mathbb{Q})$-representation $\operatorname{Pic}\left(S_{\overline{\mathbb{Q}}}\right) \otimes_{\mathbb{Z}} \mathbb{C}$ that contains the trivial representation $t$ times as a direct summand, it follows that $L\left(s, \chi_{\operatorname{Pic}\left(S_{\overline{\mathbb{Q}}}\right)}\right)=\zeta(s)^{t} \cdot L\left(s, \chi_{P}\right)$ and

$$
\lim _{s \rightarrow 1}(s-1)^{t} L\left(s, \chi_{\operatorname{Pic}\left(S_{\bar{Q}}\right)}\right)=L\left(1, \chi_{P}\right),
$$

where $\zeta$ denotes the Riemann zeta function and $P$ is a representation that does not contain trivial components. Corollaries 11.5 and 11.4 of [Murty] show that $L\left(s, \chi_{P}\right)$ has neither a pole nor a zero at $s=1$.

Finally, $\tau_{H}$ is the Tamagawa measure on the set $S\left(\mathbb{A}_{\mathbb{Q}}\right)$ of adelic points on $S$, and $S\left(\mathbb{A}_{\mathbb{Q}}\right)^{\mathrm{Br}} \subseteq S\left(\mathbb{A}_{\mathbb{Q}}\right)$ denotes the subset of the adelic points that are BrauerManin unobstructed.

Since $S$ is projective, we have

$$
S\left(\mathbb{A}_{\mathbb{Q}}\right)=\prod_{\nu \in \operatorname{Val}(\mathbb{Q})} S\left(\mathbb{Q}_{\nu}\right) .
$$

The Tamagawa measure $\tau_{H}$ is defined to be a product measure $\tau_{H}:=\prod_{\nu \in \operatorname{Val}(\mathbb{Q})} \tau_{\nu}$.

For a prime number $p$, the local measure $\tau_{p}$ is given as follows. Let $a \in S\left(\mathbb{Z} / p^{k} \mathbb{Z}\right)$ and put

$$
\mathfrak{U}_{a}^{(k)}:=\left\{x \in S\left(\mathbb{Q}_{p}\right) \mid x \equiv a\left(\bmod p^{k}\right)\right\} .
$$

Then

$$
\begin{aligned}
\tau_{p}\left(\mathfrak{U}_{a}^{(k)}\right):= & \operatorname{det}\left(1-p^{-1} \operatorname{Frob}_{p} \mid \operatorname{Pic}\left(S_{\overline{\mathbb{Q}}}\right)^{I_{p}}\right) \\
& \times \lim _{n \rightarrow \infty} \frac{\#\left\{y \in S\left(\mathbb{Z} / p^{n} \mathbb{Z}\right) \mid y \equiv a\left(\bmod p^{k}\right)\right\}}{p^{n \operatorname{dim} S}} .
\end{aligned}
$$

Here $\operatorname{Pic}\left(S_{\overline{\mathbb{Q}}}\right)^{I_{p}}$ denotes the fixed module under the inertia group.

The measure $\tau_{\infty}$ is described in [Peyre 95, Lemme 5.4.7]. In the case of a hypersurface of degree $d$ defined by the equation $f=0$, this yields

$$
\tau_{\infty}(U)=\frac{n+1-d}{2} \int_{\substack{C U \\\left|x_{0}\right|, \ldots,\left|x_{n}\right| \leq 1}} \omega_{\text {Leray }}
$$

for every Borel set $U \subset S(\mathbb{R})$. Here $\omega_{\text {Leray }}$ is the Leray measure on the cone $C S(\mathbb{R}) \subset \mathbb{R}^{n+1}$ associated to the equation $f=0$. It is given by the differential form $\frac{1}{\left|\partial f / \partial x_{0}\right|} d x_{1} \wedge \cdots \wedge d x_{n}$.

Remark 2.1. The Leray measure differs from the "surface area" that is typically introduced for hypersurfaces in $\mathbb{R}^{n+1}$ in multivariable calculus. It is related to that measure by the formula $\omega_{\text {Leray }}=\frac{1}{\|\operatorname{grad} f\|} \omega_{\text {hyp }}$.

Remark 2.2. At least for diagonal cubic surfaces, the reciprocal $\frac{1}{\tau(S)}$ admits a fundamental finiteness property. More precisely, for each $\varepsilon>0$ there exists a constant $C(\varepsilon)>0$ such that

$$
\frac{1}{\tau\left(S^{\mathfrak{a}}\right)} \geq C(\varepsilon) \cdot \mathrm{H}_{\text {naive }}\left(\frac{1}{a_{0}}: \cdots: \frac{1}{a_{3}}\right)^{\frac{1}{3}-\varepsilon}
$$

for every $\mathfrak{a} \in(\mathbb{Z} \backslash\{0\})^{4}$. In particular, there is an estimate for $\mathrm{m}(S)$ in terms of $\tau(S)$. Details on this are given in [Elsenhans and Jahnel 10b].

\section{A TECHNICAL LEMMA}

In this section, we will give estimates for the factors $\tau_{p}\left(S\left(\mathbb{Q}_{p}\right)\right)$ of Peyre's constant at the primes $p$ of good reduction. These are, in fact, regularized versions of the numbers $\# S\left(\mathbb{F}_{p}\right)$. Our main tool will be the Lefschetz trace formula.

Lemma 3.1. Good reduction: If $p \nmid 3 a_{0} \cdots a_{3}$, then the sequence $\left(\# S^{\left(a_{0}, \ldots, a_{3}\right)}\left(\mathbb{Z} / p^{n} \mathbb{Z}\right) / p^{2 n}\right)_{n \in \mathbb{N}}$ is constant.

Bad reduction:

(i) If $p$ divides $a_{0} \cdots a_{3}$ but not 3 , then the sequence

$$
\left(\# S^{\left(a_{0}, \ldots, a_{3}\right)}\left(\mathbb{Z} / p^{n} \mathbb{Z}\right) / p^{2 n}\right)_{n \in \mathbb{N}}
$$

becomes stationary as soon as $p^{n}$ does not divide any of the coefficients $a_{0}, \ldots, a_{3}$.

(ii) If $p=3$, then the sequence

$$
\left(\# S^{\left(a_{0}, \ldots, a_{3}\right)}\left(\mathbb{Z} / p^{n} \mathbb{Z}\right) / p^{2 n}\right)_{n \in \mathbb{N}}
$$


becomes stationary as soon as $3^{n}$ does not divide any of the numbers $3 a_{0}, \ldots, 3 a_{3}$.

\section{Lemma 3.2.}

(a) For every $a_{0}, \ldots, a_{3} \in \mathbb{Z} \backslash\{0\}$, the infinite product

$$
\prod_{p \text { prime }} \tau_{p}\left(S^{\left(a_{0}, \ldots, a_{3}\right)}\left(\mathbb{Q}_{p}\right)\right)
$$

is absolutely convergent.

(b) There are two positive constants $C_{1}$ and $C_{2}$ such that for all $a_{0}, \ldots, a_{3} \in \mathbb{Z} \backslash\{0\}$,

$$
C_{1}<\prod_{\substack{p \text { prime } \\ p \nmid 3 a_{0} \cdots a_{3}}} \tau_{p}\left(S^{\left(a_{0}, \ldots, a_{3}\right)}\left(\mathbb{Q}_{p}\right)\right)<C_{2} .
$$

Proof. For a prime $p$ of good reduction, Lemma 3.1 shows that

$$
\begin{aligned}
\tau_{p}\left(S^{\left(a_{0}, \ldots, a_{3}\right)}\left(\mathbb{Q}_{p}\right)\right)= & \operatorname{det}\left(1-p^{-1} \operatorname{Frob}_{p} \mid \operatorname{Pic}\left(S_{\overline{\mathbb{Q}}}\right)\right) \\
& \times \frac{\# S^{\left(a_{0}, \ldots, a_{3}\right)}\left(\mathbb{F}_{p}\right)}{p^{2}} .
\end{aligned}
$$

Further, for the number of points on a nonsingular cubic surface over a finite field, the Lefschetz trace formula can be made completely explicit [Manin 74, Theorem 27.1]. It shows that

$$
\# S^{\left(a_{0}, \ldots, a_{3}\right)}\left(\mathbb{F}_{p}\right)=p^{2}+p \cdot \operatorname{tr}\left(\operatorname{Frob}_{p} \mid \operatorname{Pic}\left(S_{\overline{\mathbb{Q}}}\right)\right)+1 .
$$

Denoting the eigenvalues of the Frobenius on $\operatorname{Pic}\left(S_{\overline{\mathbb{Q}}}\right)$ by $\lambda_{1}, \ldots, \lambda_{7}$, we obtain

$$
\begin{aligned}
\tau_{p}( & \left.S^{\left(a_{0}, \ldots, a_{3}\right)}\left(\mathbb{Q}_{p}\right)\right) \\
= & \left(1-\lambda_{1} p^{-1}\right)\left(1-\lambda_{2} p^{-1}\right) \cdots\left(1-\lambda_{7} p^{-1}\right) \\
& \times\left[1+\left(\lambda_{1}+\cdots+\lambda_{7}\right) p^{-1}+p^{-2}\right] \\
= & \left(1-\sigma_{1} p^{-1}+\sigma_{2} p^{-2} \mp \cdots-\sigma_{7} p^{-7}\right) \\
& \times\left(1+\sigma_{1} p^{-1}+p^{-2}\right) \\
= & 1+\left(1-\sigma_{1}^{2}+\sigma_{2}\right) p^{-2}-\left(\sigma_{1}-\sigma_{1} \sigma_{2}+\sigma_{3}\right) p^{-3} \pm \cdots \\
& -\left(\sigma_{5}-\sigma_{1} \sigma_{6}+\sigma_{7}\right) p^{-7}+\left(\sigma_{6}-\sigma_{1} \sigma_{7}\right) p^{-8}-\sigma_{7} p^{-9}
\end{aligned}
$$

where the $\sigma_{i}$ denote the elementary symmetric functions in $\lambda_{1}, \ldots, \lambda_{7}$.

We know that $\left|\lambda_{i}\right|=1$ for all $i$. Estimating very roughly, we have $\left|\sigma_{j}\right| \leq\left(\begin{array}{c}7 \\ j\end{array}\right) \leq 7^{j}$ and see that

$$
\begin{aligned}
1- & 99 p^{-2}-7 \cdot 99 p^{-3}-\cdots-7^{7} \cdot 99 p^{-9} \\
& \leq \tau_{p}\left(S^{\left(a_{0}, \ldots, a_{3}\right)}\left(\mathbb{Q}_{p}\right)\right) \\
& \leq 1+99 p^{-2}+7 \cdot 99 p^{-3}+\cdots+7^{7} \cdot 99 p^{-9} .
\end{aligned}
$$

That is

$$
\begin{aligned}
1-99 p^{-2} \frac{1}{1-7 / p} & <\tau_{p}\left(S^{\left(a_{0}, \ldots, a_{3}\right)}\left(\mathbb{Q}_{p}\right)\right) \\
& <1+99 p^{-2} \frac{1}{1-7 / p}
\end{aligned}
$$

The infinite product over all $1-99 p^{-2} \frac{1}{1-7 / p}$ (respectively $\left.1+99 p^{-2} \frac{1}{1-7 / p}\right)$ is convergent.

The left-hand side is positive for $p>13$. For the small primes remaining, we need a better lower bound. For this, note that a cubic surface over a finite field $\mathbb{F}_{p}$ always has at least one $\mathbb{F}_{p}$-rational point. This yields $\tau_{p}\left(S^{\left(a_{0}, \ldots, a_{3}\right)}\left(\mathbb{Q}_{p}\right)\right) \geq(1-1 / p)^{7} / p^{2}>0$.

Remark 3.3. The convergence generating factors

$$
\operatorname{det}\left(1-p^{-1} \operatorname{Frob}_{p} \mid \operatorname{Pic}\left(S_{\overline{\mathbb{Q}}}\right)^{I_{p}}\right)
$$

are all positive. Indeed, for a pair of complex conjugate eigenvalues, we have

$$
\left(1-\lambda p^{-1}\right)\left(1-\bar{\lambda} p^{-1}\right)=\left|1-\lambda p^{-1}\right|^{2}>0,
$$

and an eigenvalue of 1 or $(-1)$ contributes a factor $1 \pm$ $p^{-1}>0$. Consequently, we always have

$$
\left(1-\frac{1}{p}\right)^{7}<\operatorname{det}\left(1-p^{-1} \operatorname{Frob}_{p} \mid \operatorname{Pic}\left(S_{\overline{\mathbb{Q}}}\right)^{I_{p}}\right)<\left(1+\frac{1}{p}\right)^{7} .
$$

\section{SPLITTING THE PICARD GROUP}

\subsection{Motivation}

In the case of the diagonal cubic surface $S^{\left(a_{0}, \ldots, a_{3}\right)} \subset \mathbf{P}_{\mathbb{Q}}^{3}$, given by $a_{0} x_{0}^{3}+\cdots+a_{3} x_{3}^{3}=0$ for $a_{0}, \ldots, a_{3} \in \mathbb{Z} \backslash\{0\}$, the 27 lines on $S^{\left(a_{0}, \ldots, a_{3}\right)}$ may easily be written down explicitly. Indeed, for each pair $(i, j) \in(\mathbb{Z} / 3 \mathbb{Z})^{2}$, the system

$$
\sqrt[3]{a_{0}} x_{0}+\zeta_{3}^{i} \sqrt[3]{a_{1}} x_{1}=0, \quad \sqrt[3]{a_{2}} x_{2}+\zeta_{3}^{j} \sqrt[3]{a_{3}} x_{3}=0
$$

of equations defines a line on $S^{\left(a_{0}, \ldots, a_{3}\right)}$. Decomposing the index set $\{0, \ldots, 3\}$ differently into two subsets of two elements each yields all the lines. In particular, we see that the 27 lines may be defined over $L=\mathbb{Q}\left(\zeta_{3}, \sqrt[3]{a_{1} / a_{0}}, \sqrt[3]{a_{2} / a_{0}}, \sqrt[3]{a_{3} / a_{0}}\right)$.

It is classically known that the classes of the 27 lines on a smooth cubic surface generate its Picard group. Consequently, $\operatorname{Pic}\left(S^{\left(a_{0}, \ldots, a_{3}\right)}\right)$ is acted on by the Galois group $\operatorname{Gal}(L / \mathbb{Q})$. The goal of this section is to study the Galois module structure on $\operatorname{Pic}\left(S^{\left(a_{0}, \ldots, a_{3}\right)}\right)$ more closely. 
Fact 4.1. Let $p$ be a prime number and $a_{0}, \ldots, a_{3}$ integers not divisible by $p$. Then

$$
\begin{aligned}
& \# S^{\left(a_{0}, \ldots, a_{3}\right)}\left(\mathbb{F}_{p}\right) \\
& =\left\{\begin{array}{c}
p^{2}+\left(1+\chi_{3}\left(a_{0} a_{1} a_{2}^{2} a_{3}^{2}\right)+\chi_{3}\left(a_{0}^{2} a_{1}^{2} a_{2} a_{3}\right)\right. \\
+\chi_{3}\left(a_{0} a_{1}^{2} a_{2} a_{3}^{2}\right)+\chi_{3}\left(a_{0}^{2} a_{1} a_{2}^{2} a_{3}\right) \\
\left.\quad+\chi_{3}\left(a_{0} a_{1}^{2} a_{2}^{2} a_{3}\right)+\chi_{3}\left(a_{0}^{2} a_{1} a_{2} a_{3}^{2}\right)\right) p+1 \\
\text { if } p \equiv 1(\bmod 3), \\
p^{2}+p+1 \quad \text { if } p \equiv 2(\bmod 3) .
\end{array}\right.
\end{aligned}
$$

Here in the case $p \equiv 1(\bmod 3), \chi_{3}: \mathbb{F}_{p}^{*} \rightarrow \mathbb{C}$ denotes a cubic residue character.

Proof. If $p \equiv 2(\bmod 3)$, then every residue class modulo $p$ has a unique cube root. This immediately shows that $\# S^{\left(a_{0}, \ldots, a_{3}\right)}\left(\mathbb{F}_{p}\right)=p^{2}+p+1$.

For $p \equiv 1(\bmod 3)$, the number of $\mathbb{F}_{p}$-rational points on $S$ may be determined using Jacobi sums. The formula given follows directly from [Ireland and Rosen 82, Chapter 10, Theorem 2] together with the well-known relation $g\left(\chi_{3}\right) g\left(\chi_{3}^{2}\right)=p$ for cubic Gauss sums.

Lemma 4.2. Let $a_{0}, \ldots, a_{3} \in \mathbb{Z} \backslash\{0\}$. Then for each prime $p$ such that $p \nmid 3 a_{0} \cdots a_{3}$,

$$
\begin{aligned}
& \chi_{\operatorname{Pic}\left(S_{\overline{\mathrm{Q}}}^{\left(a_{0}, \ldots, a_{3}\right)}\right)\left(\operatorname{Frob}_{p}\right)}=\left\{\begin{array}{c}
\chi_{3}\left(a_{0} a_{1} a_{2}^{2} a_{3}^{2}\right)+\chi_{3}\left(a_{0}^{2} a_{1}^{2} a_{2} a_{3}\right)+\chi_{3}\left(a_{0} a_{1}^{2} a_{2} a_{3}^{2}\right) \\
\quad+\chi_{3}\left(a_{0}^{2} a_{1} a_{2}^{2} a_{3}\right)+\chi_{3}\left(a_{0} a_{1}^{2} a_{2}^{2} a_{3}\right)+\chi_{3}\left(a_{0}^{2} a_{1} a_{2} a_{3}^{2}\right) \\
\quad+1 \quad \text { if } p \equiv 1(\bmod 3), \\
1 \quad \text { if } p \equiv 2(\bmod 3) .
\end{array}\right.
\end{aligned}
$$

Proof. Since we have good reduction, the trace of Frob $_{p}$ on $\operatorname{Pic}\left(S_{\overline{\mathbb{Q}}}^{\left(a_{0}, \ldots, a_{3}\right)}\right) \otimes_{\mathbb{Z}} \mathbb{C}$ is the same as that of Frob on $\operatorname{Pic}\left(S_{\overline{\mathbb{F}_{p}}}^{\left(a_{0}, \overline{\mathbb{Q}}, a_{3}\right)}\right) \otimes_{\mathbb{Z}} \mathbb{C}$. Further, the Lefschetz trace formula [Manin 74, Theorem 27.1] shows that

$$
\begin{aligned}
& \# S^{\left(a_{0}, \ldots, a_{3}\right)}\left(\mathbb{F}_{p}\right) \\
& \quad=p^{2}+p \cdot \operatorname{tr}\left(\text { Frob } \mid \operatorname{Pic}\left(S_{\overline{\mathbb{F}}_{p}}^{\left(a_{0}, \ldots, a_{3}\right)}\right) \otimes_{\mathbb{Z}} \mathbb{C}\right)+1 .
\end{aligned}
$$

The explicit formulas for the numbers of points given in Fact 4.1 therefore yield the assertion.

Notation 4.3. Let $A$ be an integer, $K:=\mathbb{Q}\left(\zeta_{3}, \sqrt[3]{A}\right)$, $G:=\operatorname{Gal}(K / \mathbb{Q}), H:=\operatorname{Gal}\left(K / \mathbb{Q}\left(\zeta_{3}\right)\right)$, and $\chi: H \rightarrow \mathbb{C}^{*}$ a primitive character. Then we write $\nu^{K}:=\operatorname{ind}_{H}^{G}(\chi)$ for the induced character and $\mathbf{V}^{K}$ for the corresponding $G$-representation.
If $K$ is of degree three over $\mathbb{Q}\left(\zeta_{3}\right)$, then $\mathbf{V}^{K}$ is an irreducible rank-two representation of $G \cong \mathfrak{S}_{3}$. Otherwise, $K=\mathbb{Q}\left(\zeta_{3}\right)$. Then $\mathbf{V}^{K} \cong \mathbb{C} \oplus M$ splits into the direct sum of a trivial and a nontrivial one-dimensional representation of $H \cong \mathbb{Z} / 2 \mathbb{Z}$.

We will freely consider $\mathbf{V}^{K}$ as a $\operatorname{Gal}(\overline{\mathbb{Q}} / \mathbb{Q})$ representation.

Lemma 4.4. Let $A$ be any integer. Then for a prime $p$ not dividing $A$, we have

$\nu^{\mathbb{Q}\left(\zeta_{3}, \sqrt[3]{A}\right)}\left(\operatorname{Frob}_{p}\right)= \begin{cases}\chi_{3}(A)+\bar{\chi}_{3}(A) & \text { if } p \equiv 1(\bmod 3), \\ 0 & \text { if } p \equiv 2(\bmod 3) .\end{cases}$

Proof. The primitive character is unique up to conjugation by an element of $G$. Therefore, the induced character $\lambda$ is well defined.

The Kummer pairing allows us to make a definite choice for $\chi$ as follows. Fix an embedding $\sigma: \mathbb{Q}\left(\zeta_{3}\right) \rightarrow \mathbb{C}$. Then put $\chi(g):=\sigma(g(\sqrt[3]{A}) / \sqrt[3]{A})$.

If $p \equiv 2(\bmod 3)$, then $p$ remains prime in $\mathbb{Q}\left(\zeta_{3}\right)$. This means that Frob $_{p}$ acts nontrivially on $\mathbb{Q}\left(\zeta_{3}\right)$. That is, Frob $_{p} \in G \backslash H$. Since $H$ is a normal subgroup in $G$, the induced character vanishes on such an element.

For $p \equiv 1(\bmod 3)$, we have that $(p)$ splits in $\mathbb{Q}\left(\zeta_{3}\right)$. Let us write $(p)=\mathfrak{p} \overline{\mathfrak{p}}$. The choice of $\mathfrak{p}$ is equivalent to the choice of a homomorphism $\iota:\left\langle\zeta_{3}\right\rangle \rightarrow \mathbb{F}_{p}^{*}$. The Frobenius Frob $_{p}$ is determined only up to conjugation, and we may choose $\operatorname{Frob}_{p}=$ Frob $_{\mathfrak{p}} \in H$. Then, directly by the definition of an induced character,

$$
\nu^{\mathbb{Q}\left(\zeta_{3}, \sqrt[3]{A}\right)}\left(\operatorname{Frob}_{p}\right)=\chi\left(\text { Frob }_{\mathfrak{p}}\right)+\bar{\chi}\left(\text { Frob }_{\mathfrak{p}}\right) .
$$

We need to show that

$$
\chi\left(\operatorname{Frob}_{\mathfrak{p}}\right)=\chi_{3}(A)
$$

or

$$
\chi\left(\operatorname{Frob}_{\mathfrak{p}}\right)=\bar{\chi}_{3}(A) .
$$

For this, by the choice made above, we have

$$
\chi\left(\operatorname{Frob}_{\mathfrak{p}}\right):=\sigma\left(\operatorname{Frob}_{\mathfrak{p}}(\sqrt[3]{A}) / \sqrt[3]{A}\right) .
$$

After reduction modulo $\mathfrak{p}$, we may write

$$
\operatorname{Frob}(\sqrt[3]{A}) / \sqrt[3]{A}=(\sqrt[3]{A})^{p} / \sqrt[3]{A}=A^{\frac{p-1}{3}} .
$$

Therefore,

$$
\operatorname{Frob}_{\mathfrak{p}}(\sqrt[3]{A}) / \sqrt[3]{A}=\iota^{-1}\left(A^{\frac{p-1}{3}}\right),
$$

which shows that $\chi\left(\operatorname{Frob}_{p}\right)=\sigma\left(\iota^{-1}\left(A^{\frac{p-1}{3}}\right)\right)$. The final formula is a definition for a cubic residue character at $A$. 
Theorem 4.5. Let $a_{0}, \ldots, a_{3} \in \mathbb{Z} \backslash\{0\}$. Then the $\operatorname{Gal}(\overline{\mathbb{Q}} / \mathbb{Q})$-representation $\operatorname{Pic}\left(S_{\overline{\mathbb{Q}}}^{\left(a_{0}, \ldots, a_{3}\right)}\right) \otimes_{\mathbb{Z}} \mathbb{C}$ splits into the direct sum

$$
\operatorname{Pic}\left(S_{\overline{\mathbb{Q}}}^{\left(a_{0}, \ldots, a_{3}\right)}\right) \otimes_{\mathbb{Z}} \mathbb{C} \cong \mathbb{C} \oplus \mathbf{V}^{K_{1}} \oplus \mathbf{V}^{K_{2}} \oplus \mathbf{V}^{K_{3}}
$$

for $K_{1}:=\mathbb{Q}\left(\zeta_{3}, \sqrt[3]{a_{0} a_{1} a_{2}^{2} a_{3}^{2}}\right), K_{2}:=\mathbb{Q}\left(\zeta_{3}, \sqrt[3]{a_{0} a_{1}^{2} a_{2} a_{3}^{2}}\right)$, and $K_{3}:=\mathbb{Q}\left(\zeta_{3}, \sqrt[3]{a_{0} a_{1}^{2} a_{2}^{2} a_{3}}\right)$.

Proof. We will show that the representations on both sides have the same character. For that, by virtue of the Čebotarev density theorem, it suffices to consider the values at $\operatorname{Frob}_{p}$ for $p \nmid 3 a_{0} \cdots a_{3}$.

For the representation on the left-hand side, $\left.\chi_{\operatorname{Pic}\left(S_{\frac{Q}{Q}}\left(a_{0}, \ldots, a_{3}\right)\right.}\right)\left(\operatorname{Frob}_{p}\right)$ has been computed in Lemma 4.2. For the representation on the right-hand side, Lemma 4.4 shows that exactly the same formula is true.

Corollary 4.6. Let $a_{0}, \ldots, a_{3} \in \mathbb{Z} \backslash\{0\}$ be integers. Consider

$$
V^{\left(a_{0}, \ldots, a_{3}\right)}:=\operatorname{Pic}\left(S_{\overline{\mathbb{Q}}}^{\left(a_{0}, \ldots, a_{3}\right)}\right) \otimes_{\mathbb{Z}} \mathbb{C}
$$

as a $\operatorname{Gal}(\overline{\mathbb{Q}} / \mathbb{Q})$-representation, and let $\chi^{\left(a_{0}, \ldots, a_{3}\right)}$ be the associated character. Put $K_{1}:=\mathbb{Q}\left(\zeta_{3}, \sqrt[3]{a_{0} a_{1} a_{2}^{2} a_{3}^{2}}\right)$, $K_{2}:=\mathbb{Q}\left(\zeta_{3}, \sqrt[3]{a_{0} a_{1}^{2} a_{2} a_{3}^{2}}\right)$, and $K_{3}:=\mathbb{Q}\left(\zeta_{3}, \sqrt[3]{a_{0} a_{1}^{2} a_{2}^{2} a_{3}}\right)$. Then for the Artin conductor $N_{\chi^{\left(a_{0}, \ldots, a_{3}\right)}}$ of $\chi^{\left(a_{0}, \ldots, a_{3}\right)}$, we have

$$
N_{\chi^{\left(a_{0}, \ldots, a_{3}\right)}}^{2}=\mathbf{D}\left(K_{1}\right) \mathbf{D}\left(K_{2}\right) \mathbf{D}\left(K_{3}\right) /(-27),
$$

where

$$
\mathbf{D}(K):= \begin{cases}\operatorname{Disc}(K / \mathbb{Q}) & \text { if }\left[K: \mathbb{Q}\left(\zeta_{3}\right)\right]=3, \\ -27 & \text { if } K=\mathbb{Q}\left(\zeta_{3}\right) .\end{cases}
$$

Proof. We have to show that $N_{\nu^{K}}^{2}=\mathbf{D}(K) /(-3)$. Assume first that $\left[K: \mathbb{Q}\left(\zeta_{3}\right)\right]=3$. Then the conductordiscriminant formula [Neukirch 99, Chapter VII, Section (11.9)] shows that $\operatorname{Disc}(K / \mathbb{Q})=N_{\mathbb{C}} N_{M} N_{\nu^{K}}^{2}$ and $-3=\operatorname{Disc}\left(\mathbb{Q}\left(\zeta_{3}\right) / \mathbb{Q}\right)=N_{\mathbb{C}} N_{M}$, which together yield the assertion. In the opposite case, we have $\mathbf{V}^{K}=\mathbb{C} \oplus M$ and $N_{\nu^{K}}=N_{\mathbb{C}} N_{M}=-3$.

Lemma 4.7. Let $a$ and $b$ be integers different from zero. Then

$$
\left|\operatorname{Disc}\left(\mathbb{Q}\left(\zeta_{3}, \sqrt[3]{a b^{2}}\right) / \mathbb{Q}\right)\right| \leq 3^{9} a^{4} b^{4} .
$$

Proof. We have

$$
\begin{aligned}
& \left|\operatorname{Disc}\left(\mathbb{Q}\left(\zeta_{3}, \sqrt[3]{a b^{2}}\right) / \mathbb{Q}\right)\right| \\
& \quad \leq\left|\operatorname{Disc}\left(\mathbb{Q}\left(\zeta_{3}\right) / \mathbb{Q}\right)\right|^{3} \cdot \operatorname{Disc}\left(\mathbb{Q}\left(\sqrt[3]{a b^{2}}\right) / \mathbb{Q}\right)^{2} \\
& \quad=27 \cdot \operatorname{Disc}\left(\mathbb{Q}\left(\sqrt[3]{a b^{2}}\right) / \mathbb{Q}\right)^{2} .
\end{aligned}
$$

Further, by [Marcus 77, Chapter 2, Exercise 41], we know that $\left|\operatorname{Disc}\left(\mathbb{Q}\left(\sqrt[3]{a b^{2}}\right) / \mathbb{Q}\right)\right| \leq 3^{3} a^{2} b^{2}$. This shows that $\left|\operatorname{Disc}\left(\mathbb{Q}\left(\zeta_{3}, \sqrt[3]{a b^{2}}\right) / \mathbb{Q}\right)\right| \leq 3^{9} a^{4} b^{4}$.

Corollary 4.8. Let $a_{0}, \ldots, a_{3} \in \mathbb{Z} \backslash\{0\}$ be integers and $\chi^{\left(a_{0}, \ldots, a_{3}\right)}$ the character associated to the $\mathrm{Gal}(\overline{\mathbb{Q}} / \mathbb{Q})$ representation

$$
V^{\left(a_{0}, \ldots, a_{3}\right)}:=\operatorname{Pic}\left(S_{\overline{\mathbb{Q}}}^{\left(a_{0}, \ldots, a_{3}\right)}\right) \otimes_{\mathbb{Z}} \mathbb{C} .
$$

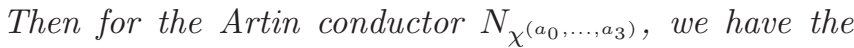
estimate

$$
\left|N_{\chi^{\left(a_{0}, \ldots, a_{3}\right)}}\right| \leq 3^{12}\left(a_{0} \cdots a_{3}\right)^{6} .
$$

Proof. Lemma 4.7 shows that $\left|\mathbf{D}\left(K_{i}\right)\right| \leq 3^{9}\left(a_{0} \cdots a_{3}\right)^{4}$ for $i=1,2,3$, from which the assertion follows immediately.

\section{THE COMPUTATION OF THE $L$-FUNCTION AT 1}

We now return to the particular diagonal cubic surfaces treated in the numerical experiment; cf. Section 1 for a description of our sample.

Lemma 5.1. For $a, b \in \mathbb{Z} \backslash\{0\}$, consider in $\mathbf{P}_{\mathbb{Q}}^{3}$ the diagonal cubic surface $S=S^{(a, b, 2,1)}$. Assume that $S$ satisfies condition (i), (ii), or (iii) of Section 1.

(i) Then $\operatorname{rk} \operatorname{Pic}(S)=1$.

(ii) Furthermore, we have the relation

$$
\begin{aligned}
& \lim _{s \rightarrow 1}(s-1) L\left(s, \chi_{\operatorname{Pic}\left(S_{\bar{\Phi}}\right)}\right) \\
& \quad=L\left(1, \nu^{K_{1}}\right) L\left(1, \nu^{K_{2}}\right) L\left(1, \nu^{K_{3}}\right)
\end{aligned}
$$

for $K_{1}=\mathbb{Q}\left(\zeta_{3}, \sqrt[3]{4 a b}\right), K_{2}=\mathbb{Q}\left(\zeta_{3}, \sqrt[3]{2 a b^{2}}\right)$, and $K_{3}=\mathbb{Q}\left(\zeta_{3}, \sqrt[3]{4 a b^{2}}\right)$.

Proof. (i) The assumptions imply that $4 a b, 2 a b^{2}$, and $4 a b^{2}$ are three noncubes. In particular, the $\operatorname{Gal}(\overline{\mathbb{Q}} / \mathbb{Q})$ representations $\mathbf{V}^{K_{1}}, \mathbf{V}^{K_{2}}$, and $\mathbf{V}^{K_{3}}$ are irreducible of rank two.

Further, a standard application of the Hochschild-Serre spectral sequence ensures that $\operatorname{Pic}(S) \subseteq \operatorname{Pic}\left(S_{\overline{\mathbb{Q}}}\right)^{\operatorname{Gal}(\overline{\mathbb{Q}} / \mathbb{Q})}$ is always a subgroup of finite index. Therefore, it suffices to verify that $\operatorname{rk} \operatorname{Pic}\left(S_{\overline{\mathbb{Q}}}\right) \operatorname{Gal}(\overline{\mathbb{Q}} / \mathbb{Q})=1$. For this, we note that by Theorem 4.5, $\operatorname{Pic}\left(S_{\overline{\mathbb{Q}}}\right)^{\operatorname{Gal}(\overline{\mathbb{Q}} / \mathbb{Q})} \otimes_{\mathbb{Z}} \mathbb{C}$ splits into a trivial and three irreducible $\mathrm{Gal}(\overline{\mathbb{Q}} / \mathbb{Q})$-representations.

(ii) Note again that $\chi_{\operatorname{Pic}\left(S_{\overline{\mathbb{Q}}}\right)}=1+\nu^{K_{1}}+\nu^{K_{2}}+\nu^{K_{3}}$. The assertion follows directly from [Neukirch 99, Chapter VII, Theorem (10.4).(ii)]. 
We make the following observations:

(i) The character $\nu^{K_{i}}$ is induced by a nontrivial character of the group $\operatorname{Gal}\left(K_{i} / \mathbb{Q}\left(\zeta_{3}\right)\right)$ of order three. Therefore, by [Neukirch 99, Chapter VII, Theorem (10.4.iv)], we may understand $L\left(s, \nu^{K_{i}}\right)$ as the Artin $L$-function over $\mathbb{Q}\left(\zeta_{3}\right)$ associated to that character.

(ii) Further, $K_{i} / \mathbb{Q}\left(\zeta_{3}\right)$ is an abelian extension. Then [Neukirch 99, Chapter VII, Theorem (10.6)] shows that $L\left(s, \nu^{K_{i}}\right)$ coincides with the Hecke $L$-function given by the generalized Dirichlet character of order three modulo $4 a b, 2 a b^{2}$, or $4 a b^{2}$ over $\mathbb{Q}\left(\zeta_{3}\right)$. An elementary proof of this fact requires the cubic reciprocity law [Ireland and Rosen 82].

Remark 5.2. Since $L\left(1, \nu^{K_{i}}\right)$ is not given by an absolutely convergent series, we cannot evaluate it directly.

Remark 5.3. One could apply the analytic class number formula to compute $L\left(1, \nu^{K_{i}}\right)$. This approach is, however, not practical for half a million $L$-functions.

Notation 5.4. From now on, we will denote the generalized Dirichlet character of order three modulo $A$ by $\nu_{A}$ and its conductor by $m \in \mathbb{Z}\left[\zeta_{3}\right]$. Further, we write $N: \mathbb{Q}\left(\zeta_{3}\right) \rightarrow \mathbb{Q}$ for the norm map.

We complete the $L$-function by putting

$$
\Lambda\left(s, \nu_{A}\right):=(-3 N(m))^{s / 2} \frac{2}{(2 \pi)^{s}} \Gamma(s) L\left(s, \nu_{A}\right) .
$$

The completed $L$-function is connected with a theta function via a Mellin transform. One has

$$
\Lambda\left(s, \nu_{A}\right)=\int_{0}^{\infty} f(t) t^{s / 2} \frac{d t}{t}
$$

where $f$ is the function defined by

$$
f(t):=\frac{1}{6} \sum_{a \in \mathbb{Z}\left[\zeta_{3}\right]} \nu_{A}(a) e^{-\frac{2 \pi}{|3 m|} N(a) \sqrt{t}}
$$

for $t>0$. The connection to the Hecke theta function associated to $\mathbb{Z}\left[\zeta_{3}\right]$ and $\nu_{A}$ is given by

$$
f(t):=\frac{1}{6} \theta\left(i \sqrt{t}, \nu_{A}\right) .
$$

Inspecting the convergence properties of the series, we see that it converges very rapidly for $t \gg 0$, while convergence is arbitrarily slow for $t$ close to zero.
The functional equation

$$
\theta\left(-1 / z, \nu_{A}\right)=\frac{z}{i} \theta\left(z, \bar{\nu}_{A}\right)
$$

interchanges the ranges of good and bad convergence. Hence this equation should be used to compute $f(t)$ for $t$ small.

To be more precise, we split the half-line $[0, \infty)$ into two parts and write

$$
\Lambda\left(s, \nu_{A}\right)=\int_{0}^{u} f(t) t^{s / 2} \frac{d t}{t}+\int_{u}^{\infty} f(t) t^{s / 2} \frac{d t}{t} .
$$

Applying the functional equation of the Hecke theta function to the first summand yields

$$
\begin{aligned}
& \Lambda\left(s, \nu_{A}\right) \\
& =\frac{1}{6} \sum_{a \in \mathbb{Z}\left[\zeta_{3}\right]} 2 \nu_{A}(a)\left(\left[\frac{|3 m|}{2 \pi N(a)}\right]^{1-s} \int_{\frac{2 \pi N(a)}{|3 m|} \frac{1}{\sqrt{u}}}^{\infty} e^{-x} x^{-s} d x\right. \\
& \left.\quad+\left[\frac{|3 m|}{2 \pi N(a)}\right]^{s} \int_{\frac{2 \pi N(a)}{|3 m|} \sqrt{u}}^{\infty} e^{-x} x^{s-1} d x\right)
\end{aligned}
$$

for each $u>0$. This is an absolutely convergent infinite series.

Remark 5.5. The idea to evaluate an $L$-function at an arbitrary point $s \in \mathbb{C}$ using a series analogous to (5-1) goes back at least to [Lavrik 67]. Descriptions of similar methods may also be found in [Stark 75], [Cohen 00, Section 10.3], and [Dokchitser 04].

Remark 5.6. The relation of $\Lambda\left(s, \nu_{A}\right)$ to a theta function is a particular case of the very general [Neukirch 99, Chapter VII, Theorem (8.3)]. In comparison with the general case, many simplifications do occur, mainly because $\mathbb{Q}\left(\zeta_{3}\right)$ is an imaginary quadratic number field of class number 1 . Note that $\mathbb{Q}\left(\zeta_{3}\right)$ has discriminant $(-3)$ and precisely six units.

Remark 5.7. In more generality, the functional equation of a Hecke theta function is of the form

$$
\theta(-1 / z, \nu)=\frac{\tau(\nu)}{\sqrt{N(m)}} \frac{z}{i} \theta(z, \bar{\nu}) .
$$

Here, $\tau(\nu)$ is the Gauss sum associated to the character $\nu$ [Neukirch 99, Chapter VII, Definition (7.4)].

In our case, it is immediate from the definition that $\tau\left(\nu_{A}\right)$ is real. Further, [Neukirch 99, Chapter VII, Theorem (7.7)] shows that $\left|\tau\left(\nu_{A}\right)\right|=\sqrt{N(m)}$, so that the coefficient of the functional equation is \pm 1 . 


\begin{tabular}{|c|c|c|c|}
\hline Radicand $A$ & $L\left(1, \nu_{A}\right)$ using $u=1$ & $L\left(1, \nu_{A}\right)$ using $u=1.2$ & $\mathbf{L}\left(1, \nu_{A}\right)$ using class number formula \\
\hline 166,249 & 4.419173379082995 & 4.419173379082997 & 4.419173379082996519114130 \\
\hline $102,044,100$ & 0.596117703616924 & 0.596117703616918 & 0.596117703616923884079232 \\
\hline $3,586,804$ & 0.888154374767605 & 0.888154374767607 & 0.888154374767604963111775 \\
\hline $536,227,198$ & 0.946251759020570 & 0.946251759020576 & 0.946251759020569971686643 \\
\hline $1,072,454,396$ & 1.437503627427445 & 1.437503627427447 & 1.437503627427445188453952 \\
\hline
\end{tabular}

TABLE 1. Some values of the $L$-functions at $s=1$.

Actually, the sign is always positive. Indeed, a direct calculation shows that

$$
\zeta_{\mathbb{Q}(\sqrt[3]{A})}(s)=L\left(s, \nu_{A}\right) \zeta(s) .
$$

Further, in the functional equation of the Dedekind zeta function, the sign is always positive [Neukirch 99, Chapter VII, Corollary (5.10)].

Remark 5.8. The convergence of the series (5-1) is optimal when $u$ is close to 1 . Calculations using different values of $u$ may be used for checks [Dokchitser 04].

Remark 5.9. The number of summands required for a numerical approximation is about $C|m|$. The constant $C$ depends on the precision required.

Remark 5.10. There are several obvious ideas to optimize the computations:

(i) The summand for $a$ depends only on the ideal $(a)$. Hence, the summands arise in groups of six. We calculate only once for each group.

(ii) Both integrals depend only on $N(a)$ and $|m|$. Thus, we evaluate them only once for each pair $(N(a),|m|)$.

(iii) The computation of the generalized Dirichlet characters $\nu_{A}$ is speeded up using their multiplicativity in $A$. For a concrete value $a \in \mathbb{Z}\left[\zeta_{3}\right]$, we first use Euler's criterion to compute $\nu_{p}(a)$ for all prime numbers $p$ less than 3000. These values having been tabulated, the calculation of all the characters $\nu_{A}$ at $a$ is done rapidly.

Since we are interested in the evaluation of many $L$ functions at $s=1$, some further possibilities for optimization arise:

(iv) The first integral is in fact the integral exponential function, and the second one is just an exponential function. The numerical evaluation of the integral exponential function could be done by a combination of the power series expansion with a continued fraction expansion [Press et al. 86].
However, there is another method that is better. The arguments of the integral exponential function we meet lie in a rather small range. This range was split up into even smaller intervals. On each interval, we used a polynomial approximation.

We organized the computations as follows. In a first step, we enumerated all the radicands $A$ for which $L\left(1, \nu_{A}\right)$ had to be computed. We sorted the list and eliminated all repetitions. In addition, for each radicand, we stored its prime decomposition for later use. The resulting list consisted of 557,270 radicands. Only 214,285 different conductors occurred.

Then we evaluated $L\left(1, \nu_{A}\right)$ for all the radicands $A$ that occurred. We used formula (5-1) for $u=1$ and $u=1.2$. To evaluate the series numerically, we worked with 64-bit hardware floats and used backward summation. The differences between the two results were always negligible. The entire computation of the values of $L$ took around four days on a $2.2-\mathrm{GHz}$ Opteron processor.

In Table 1, we present a few of the values computed. The first two lines represent the absolutely largest and the absolutely smallest values of $L$ that we found. The other three lines correspond to conductor 5,380,206, which is the largest conductor appearing in our list. For this maximal conductor, we worked in the summation with all $a \in \mathbb{Z}\left[\zeta_{3}\right]$ such that $N(a) \leq 38,276,797$. For smaller conductors, according to Remark 5.9, fewer summands were used.

\section{COMPUTING THE TAMAGAWA NUMBERS}

Lemma 6.1. For $a, b \in \mathbb{Z} \backslash\{0\}$, consider in $\mathbf{P}_{\mathbb{Q}}^{3}$ the diagonal cubic surface $S=S^{(a, b, 2,1)}$. Assume that $S$ satisfies condition (i), (ii), or (iii) of Section 1.

(i) Then $\alpha(S)=1$ and $\beta(S)=3$.

(ii) Furthermore, one has precisely

$$
\tau_{H}\left(S\left(\AA_{\mathbb{Q}}\right)^{\mathrm{Br}}\right)=\frac{1}{3} \tau_{H}\left(S\left(\mathrm{~A}_{\mathbb{Q}}\right)\right) .
$$


Proof. (i) On a cubic surface, the self-intersection number of the canonical divisor $K$ is equal to 3 , which is square-free. Therefore, $\operatorname{rk} \operatorname{Pic}(S)=1$ immediately implies that $\operatorname{Pic}(S)=\langle K\rangle$. This is enough to ensure that $\alpha(S)=1$.

The value $\beta(S)$ can be computed using the method described in [Manin 74, Proposition 31.3]. Let $F \subset \operatorname{Div}(S)$ be the free abelian group over the 27 lines, $F_{0} \subset F$ the subset of principal divisors, and $N: F \rightarrow F$ the norm map under the operation of the Galois group $G$ on $F$. Then Manin states that

$H^{1}\left(\operatorname{Gal}(\overline{\mathbb{Q}} / \mathbb{Q}), \operatorname{Pic}\left(S_{\overline{\mathbb{Q}}}\right)\right) \cong \operatorname{Hom}\left(\left(N F \cap F_{0}\right) / N F_{0}, \mathbb{Q} / \mathbb{Z}\right)$.

We have a group $G$ of order 6,18 , or 54 . If $\# G=54$, then $G$ decomposes the 27 lines into three orbits of nine lines each. In this case, an easy calculation shows that

$$
\operatorname{Hom}\left(\left(N F \cap F_{0}\right) / N F_{0}, \mathbb{Q} / \mathbb{Z}\right) \cong \mathbb{Z} / 3 \mathbb{Z} \text {. }
$$

The smaller groups might lead to the decomposition type $[3,6,9,9]$ or $[3,3,3,6,6,6]$. A calculation in GAP shows that $\operatorname{Hom}\left(\left(N F \cap F_{0}\right) / N F_{0}, \mathbb{Q} / \mathbb{Z}\right) \cong \mathbb{Z} / 3 \mathbb{Z}$ in these cases, too.

(ii) This assertion is known from [Colliot-Thélène et al. 87, Proof of Proposition 2].

Corollary 6.2. For $a, b \in \mathbb{Z} \backslash\{0\}$, consider the diagonal cubic surface $S=S^{(a, b, 2,1)}$. Assume that $S$ satisfies condition (i), (ii), or (iii) of Section 1.

Then, for E. Peyre's Tamagawa-type number, one has

$$
\begin{aligned}
\tau(S)= & \lim _{s \rightarrow 1}(s-1) L\left(s, \chi_{\operatorname{Pic}\left(S_{\overline{\mathbb{Q}}}\right)}\right) \\
& \times \prod_{p \text { prime }} \tau_{p}\left(S\left(\mathbb{Q}_{p}\right)\right) \cdot \tau_{\infty}(S(\mathbb{R})) .
\end{aligned}
$$

\subsection{The Factor at the Infinite Place}

Since $S$ is a diagonal cubic surface, the projection from the cone $C S(\mathbb{R})$ to the $(y, z, w)$-space is one-to-one. Therefore,

$$
\begin{aligned}
\tau_{\infty} & (S(\mathbb{R})) \\
& =\frac{1}{6 \sqrt[3]{a}} \iiint_{\substack{(y, z, w) \in[-1,1]^{3} \\
|x(y, z, w)| \leq 1}} \frac{1}{\left(b y^{3}+2 z^{3}+w^{3}\right)^{2 / 3}} d y d z d w .
\end{aligned}
$$

Further, we have

$$
\begin{aligned}
|x(y, z, w)| & =\sqrt[3]{\frac{\left|b y^{3}+2 z^{3}+w^{3}\right|}{a}} \\
& \leq \sqrt[3]{\frac{b|y|^{3}+2|z|^{3}+|w|^{3}}{a}} .
\end{aligned}
$$

Since $|y| \leq 1,|z| \leq 1,|w| \leq 1$, and $a>b+3$, it turns out that the condition $|x(y, z, w)| \leq 1$ is actually empty. The integral in the formula for $\tau_{\infty}(S(\mathbb{R}))$ depends only on $b$. We are left with just 300 different integrals.

A linear substitution leads to 300 integrals of the same function on an increasing sequence of integration domains. Hence, this sequence can be computed incrementally. Doing this, the first integrals (for $b=1,2$, and 3 ) are critical, since the integrand is singular in the domain of integration. Thus, they should not be computed naively. We evaluated them using the approach described in [Elsenhans and Jahnel 10a].

\subsection{Computation of the Euler Product}

By Lemma 3.2, the Euler product is absolutely convergent, and for the relative error, we have the estimate

$$
\begin{aligned}
& \left|\prod_{\substack{p \geq N \\
p \equiv 1(\bmod 3)}}\left(1 \pm 99 p^{-2} \frac{1}{1-7 / p}\right) \cdot \prod_{\substack{p \geq N \\
p \equiv 2(\bmod 3)}}\left(1-\frac{1}{p^{3}}\right)-1\right| \\
& \quad \leq \frac{99 / 2}{N \log N}+O\left(\frac{1}{N \log ^{2} N}\right)
\end{aligned}
$$

if all bad primes are below $N$. In particular, the approximation by the finite product over all primes up to $10^{6}$ leads to a relative error of less than $4 \cdot 10^{-6}$.

The computation of the Euler products was done according to their definition. An optimization that is worth a mention is that we ran the outer loop over the prime numbers and the inner loops over $a$ and $b$. The whole computation of the Euler products took a quarter of an hour.

\section{SEARCHING FOR THE SMALLEST SOLUTION}

We will now explain how we generated the data for Figure 1. In addition to computing the Tamagawa-type numbers, we had to find the points of smallest height, i.e., the smallest solutions of the equations

$$
a x^{3}+b y^{3}+2 z^{3}+w^{3}=0,
$$

where $a=1, \ldots, 3000$ and $b=1, \ldots, 300$ satisfy the conditions (i), (ii), (iii) formulated in Section 1.

We applied a modification of the strategy from [Colliot-Thélène et al. 87, pp. 79-80]. The algorithms used are slight modifications of [Elsenhans and Jahnel 06, Algorithm 27]. We dealt with the decoupling $a x^{3}+2 z^{3}=-b y^{3}-w^{3}$. 


\subsection{Description of the Method}

Our method comprises the following steps:

(i) In a first stage, we worked with a search bound of 100 and ran the algorithm simultaneously on all the 900,000 equations for $a=1, \ldots, 3000$ and $b=1, \ldots, 300$. For exactly 69,074 of these equations, no solution was found. Among them, 67,787 satisfied the congruence conditions (i)-(iii) formulated in Section 1. In this list, there were only a few duplications. Altogether, 65,314 of the equations also obeyed the limitation $a>b+3$.

For these, we ran a test for $p$-adic solvability. It turned out that only 18,424 of the remaining 65,314 equations were solvable in $\mathbb{Q}_{p}$ for every prime $p$.

(ii) We executed the second stage with the corresponding pairs. They were read from a file. The search algorithm was run separately for each equation. We worked with search bounds of 200, 400, and 800 and stopped when a solution was found. Only 113 equations remained unsolved by that stage.

(iii) In most of these cases, there was a prime $p$ such that 2 is a cubic nonresidue modulo $p$ dividing both $a$ and $b$. This enforces that both $z$ and $w$ must be divisible by $p$. We used these strong divisibility conditions when working with search bounds of 4000 and 20,000.

Remark 7.1. In the last stage, there were only three equations remaining for which no solution had been found with a search bound of $B=4000$. They are represented by the pairs $(a, b)=(2321,211),(2331,222)$, and $(2641,278)$. The corresponding smallest solutions are respectively

$$
(-125,-884,4220,-211), \quad(-389,64,4033,1813),
$$

and

$$
(-1023,-458,11259,-695) \text {. }
$$

Remark 7.2. Altogether, there are exactly 849,781 cubic surfaces satisfying the congruence conditions and limitations given in Section 1. It turned out that 46,890 of them are $p$-adically unsolvable for some prime $p \equiv 1(\bmod 3)$. Each of the remaining cubic surfaces admits a $\mathbb{Q}$-rational point.

Thus, there are no counterexamples to the Hasse principle in our sample. This confirms the conjecture [ColliotThélène and Sansuc 81, Conjecture C].

Remark 7.3. It should be noted that [Elsenhans and Jahnel 06, Algorithm 27] itself would not work very well in this problem, at least not in the first stage. The point is that there are some numbers that appear as values of the expressions $a x^{3}+2 z^{3}$ and $\left(-b y^{3}-w^{3}\right)$ many times. Whether we chose one side or the other, we had a hash function that was quite far from being uniform.

Our idea to overcome this difficulty was to replace hashing by sorting. We generate sorted lists of all values taken by the expressions on the two sides. We look for coincidences by a procedure similar to a step of the sorting algorithm merge sort.

\section{A NEGATIVE RESULT}

In this section, we will show that the inequality $\mathrm{m}(S)<$ $C / \tau(S)$ is false, in general. We will construct a sequence $\left\{S^{(q)}\right\}_{q \in \mathbb{N}}$ of diagonal cubic surfaces such that $\mathrm{m}\left(S^{(q)}\right) \tau\left(S^{(q)}\right)$ is unbounded.

For an integer $q \neq 0$, denote by $S^{(q)} \subset \mathbf{P}_{\mathbb{Q}}^{3}$ the cubic surface given by $q x^{3}+4 y^{3}+2 z^{3}+w^{3}=0$ and let

$$
\begin{aligned}
& \operatorname{m}\left(S^{(q)}\right) \\
& :=\min \left\{\mathrm{H}_{\text {naive }}(x: y: z: w) \mid(x: y: z: w) \in S^{(q)}(\mathbb{Q})\right\}
\end{aligned}
$$

be the smallest height of a $\mathbb{Q}$-rational point on $S^{(q)}$. We want to compare $\mathrm{m}\left(S^{(q)}\right)$ with the Tamagawa-type number $\tau^{(q)}:=\tau\left(S^{(q)}\right)$.

Theorem 8.1. Assume the generalized Riemann hypothesis. Then there is no constant $C$ such that

$$
\mathrm{m}\left(S^{(q)}\right)<\frac{C}{\tau^{(q)}}
$$

for all $q \in \mathbb{Z} \backslash\{0\}$.

Proof. We will construct a sequence $\left\{q_{i}\right\}_{i \in \mathbb{N}}$ of primes such that $q_{i} \equiv 1(\bmod 72)$ and $\mathrm{m}\left(S^{\left(q_{i}\right)}\right) \tau^{\left(q_{i}\right)} \rightarrow \infty$ for $i \rightarrow \infty$. The proof will consist of several steps.

First step. It is sufficient to verify that

$$
\begin{aligned}
& \mathrm{m}\left(S^{\left(q_{i}\right)}\right) \lim _{s \rightarrow 1}(s-1) L\left(s, \chi_{\operatorname{Pic}\left(S_{\overline{\mathbb{Q}}}^{\left(q_{i}\right)}\right)}\right) \\
& \quad \times \prod_{p \text { prime }} \tau_{p}\left(S^{\left(q_{i}\right)}\left(\mathbb{Q}_{p}\right)\right) \tau_{\infty}\left(S^{\left(q_{i}\right)}(\mathbb{R})\right) \rightarrow \infty .
\end{aligned}
$$

Since $q_{i} \equiv 1(\bmod 72)$, the prime $q_{i}$ is odd. Hence, the surface $S$ satisfies condition (ii) of Section 1. The claim follows directly from Corollary 6.2.

Second step. For the height of the smallest point, we have $\mathrm{m}\left(S^{(q)}\right) \geq \sqrt[3]{\frac{q}{7}}$.

There are no rational solutions of the equation $4 y^{3}+$ $2 z^{3}+w^{3}=0$, since this is impossible 2 -adically. The inequality $|x| \geq 1$ yields $\left|4 y^{3}+2 z^{3}+w^{3}\right| \geq q$ and $\max \{|y|,|z|,|w|\} \geq \sqrt[3]{\frac{q}{7}}$. 
Third step. For $|q| \geq 7$, one has

$$
\tau_{\infty}\left(S^{(q)}(\mathbb{R})\right)=\frac{1}{\sqrt[3]{|q|}} I
$$

where $I$ is independent of $q$. This was proved in Section 6 . Fourth step. There is a positive constant $C$ such that

$$
\prod_{p \text { prime }} \tau_{p}\left(S^{(q)}\left(\mathbb{Q}_{p}\right)\right)>C
$$

for every prime $q \equiv 1(\bmod 72)$.

By Lemma 3.2 , we have $C_{1}>0$ such that

$$
\prod_{\substack{p \text { prime } \\ p \neq 2,3, q}} \tau_{p}\left(S^{(q)}\left(\mathbb{Q}_{p}\right)\right)>C_{1} .
$$

It therefore remains to give lower bounds for the factors $\tau_{2}\left(S^{(q)}\left(\mathbb{Q}_{2}\right)\right), \tau_{3}\left(S^{(q)}\left(\mathbb{Q}_{3}\right)\right)$, and $\tau_{q}\left(S^{(q)}\left(\mathbb{Q}_{q}\right)\right)$.

Since $2 \nmid q$, by virtue of Lemma 3.1 we have

$$
\tau_{2}\left(S^{(q)}\left(\mathbb{Q}_{2}\right)\right)=\frac{1}{2^{7}} \cdot \frac{\# S^{(q)}(\mathbb{Z} / 8 \mathbb{Z})}{64} .
$$

Further, $\# S^{(q)}(\mathbb{Z} / 8 \mathbb{Z}) \geq 1$, since $q \equiv 1(\bmod 8)$ implies $(1: 0: 0:(-1)) \in S^{(q)}(\mathbb{Z} / 8 \mathbb{Z})$.

Similarly,

$$
\tau_{3}\left(S^{(q)}\left(\mathbb{Q}_{3}\right)\right)=\left(\frac{2}{3}\right)^{7} \cdot \frac{\# S^{(q)}(\mathbb{Z} / 9 \mathbb{Z})}{81} .
$$

Again, $q \equiv 1(\bmod 9)$ ensures that

$$
(1: 0: 0:(-1)) \in S^{(q)}(\mathbb{Z} / 9 \mathbb{Z})
$$

and $\# S^{(q)}(\mathbb{Z} / 9 \mathbb{Z}) \geq 1$.

For the prime $q$ we argue a bit differently. First,

$$
\begin{aligned}
\operatorname{det}\left(1-q^{-1} \operatorname{Frob}_{p} \mid \operatorname{Pic}\left(S_{\frac{(q)}{\mathbb{Q}}}\right)^{I_{q}}\right) & \geq\left(1-\frac{1}{q}\right)^{7} \\
& \geq\left(\frac{72}{73}\right)^{7} .
\end{aligned}
$$

Furthermore, the reduction of $S^{(q)}$ modulo $q$ is the cone over the elliptic curve given by $4 y^{3}+2 z^{3}+w^{3}=0$. Therefore, on $S^{(q)}$ there are at least $(q-2 \sqrt{q}+1)(q-1)$ smooth points defined over $\mathbb{F}_{q}$.

Since Hensel's lemma may be applied to them, we get

$$
\begin{aligned}
\lim _{n \rightarrow \infty} \frac{\# S^{(q)}\left(\mathbb{Z} / q^{n} \mathbb{Z}\right)}{q^{2 n}} & \geq \frac{(q-2 \sqrt{q}+1)(q-1)}{q^{2}} \\
& >\left(1-\frac{2}{\sqrt{q}}\right)\left(1-\frac{1}{q}\right) \\
& \geq \frac{72}{73}\left(1-\frac{2}{\sqrt{73}}\right) .
\end{aligned}
$$

Fifth step. There is a sequence $\left\{q_{i}\right\}_{i \in \mathbb{N}}$ of primes such that $q_{i} \equiv 1(\bmod 72)$ and

$$
\left[\lim _{s \rightarrow 1}(s-1) L\left(s, \chi_{\operatorname{Pic}}\left(S_{\overline{\mathbb{Q}}}^{\left(q_{i}\right)}\right)\right)\right] \rightarrow \infty
$$

for $i \rightarrow \infty$.

Since $\operatorname{rk} \operatorname{Pic}\left(S^{\left(q_{i}\right)}\right)=1$, the representation

$$
\operatorname{Pic}\left(S_{\overline{\mathbb{Q}}}^{\left(q_{i}\right)}\right) \otimes_{\mathbb{Z}} \mathbb{C}
$$

contains exactly one trivial summand. Hence

$$
L\left(s, \chi_{\operatorname{Pic}\left(S_{\overline{\mathbb{Q}}}^{\left(q_{i}\right)}\right)}\right)=\zeta(s) \cdot L\left(s, \chi_{0}^{\left(q_{i}\right)}\right)
$$

for $\chi_{0}^{\left(q_{i}\right)}$ the character of a representation $V_{0}^{\left(q_{i}\right)}$ not containing trivial components. Our goal is therefore to show that $L\left(1, \chi_{0}^{\left(q_{i}\right)}\right) \rightarrow \infty$ for $i \rightarrow \infty$.

For each $i \in \mathbb{N}$, denote by $P_{i}$ the $i$ th prime number $p$ such that $p \equiv 1(\bmod 3)$.

We define $q_{i}$ to be the smallest prime such that

$$
q_{i} \equiv 1\left(\bmod 72 P_{1} \cdots P_{i}\right)
$$

From this, we clearly have that $q_{i}>72 P_{1} \cdots P_{i} \rightarrow \infty$ for $i \rightarrow \infty$.

Furthermore, by Chebyshev, we know that

$$
72 P_{1} \cdots P_{i} \leq 72 e^{\theta\left(P_{i}\right)}<72 e^{(2 \log 2) P_{i}} .
$$

Hence, Linnik's theorem in the version of [HeathBrown 92] shows that

$$
q_{i} \leq C_{1} \cdot\left(72 e^{(2 \log 2) P_{i}}\right)^{5.5}=C_{2} e^{(11 \log 2) P_{i}}
$$

for certain constants $C_{1}$ and $C_{2}$.

Corollary 4.8 gives us an estimate for the Artin conductor of the character $\chi^{\left(q_{i}, 4,2,1\right)}$ that is the same as that of $\chi_{0}^{\left(q_{i}\right)}$. We see that

$$
N_{\chi_{0}^{\left(q_{i}\right)}} \leq 3^{12}\left(a_{0} \cdots a_{3}\right)^{6}=3^{12} 8^{6} q_{i}^{6} \leq C_{3} e^{(66 \log 2) P_{i}}
$$

for another constant $C_{3}$.

Consequently,

$$
\log N_{\chi_{0}^{\left(q_{i}\right)}} \leq(66 \log 2) P_{i}+\log C_{3} .
$$

We observe that $\left(\log N_{\chi_{0}^{\left(q_{i}\right)}}\right)^{1 / 2} \leq P_{i}$ for $i$ sufficiently large. We assume from now on that this inequality is satisfied.

Recall from Theorem 4.5 that $V_{0}^{\left(q_{i}\right)}$ is actually the direct sum of representations that are induced from onedimensional characters. In consequence, it is known that the Artin $L$-function $L\left(\cdot, \chi_{0}^{\left(q_{i}\right)}\right)$ is entire. Since we also 
assume the generalized Riemann hypothesis, we may apply the estimate of [Duke 03, Proposition 5], which shows that

$$
\log L\left(1, \chi_{0}^{\left(q_{i}\right)}\right)=\sum_{p<\left(\log N_{\chi_{0}^{\left(q_{i}\right)}}\right)^{1 / 2}} \chi_{0}^{\left(q_{i}\right)}\left(\mathrm{Frob}_{p}\right) p^{-1}+O(1) .
$$

Here

$$
\chi_{0}^{\left(q_{i}\right)}\left(\operatorname{Frob}_{p}\right)=\chi_{\operatorname{Pic}\left(S_{\bar{Q}}^{\left(q_{i}\right)}\right)}\left(\operatorname{Frob}_{p}\right)-1 .
$$

For $p \equiv 2(\bmod 3)$, this yields $\chi_{0}^{\left(q_{i}\right)}\left(\right.$ Frob $\left._{p}\right)=0$.

On the other hand, for $p \equiv 1(\bmod 3)$, we have, by virtue of Lemma 4.2,

$$
\begin{aligned}
\chi_{0}^{\left(q_{i}\right)}\left(\operatorname{Frob}_{p}\right)= & \chi_{3}(16 q)+\chi_{3}\left(32 q^{2}\right)+\chi_{3}(32 q)+\chi_{3}\left(16 q^{2}\right) \\
& +\chi_{3}(64 q)+\chi_{3}\left(8 q^{2}\right) \\
= & \chi_{3}(q)+\chi_{3}(2 q)+\chi_{3}(4 q)+\chi_{3}\left(q^{2}\right) \\
& +\chi_{3}\left(2 q^{2}\right)+\chi_{3}\left(4 q^{2}\right) \\
= & \left(1+\chi_{3}(2)+\chi_{3}(4)\right)\left(\chi_{3}(q)+\chi_{3}\left(q^{2}\right)\right) .
\end{aligned}
$$

This may be written down in an explicit form as

$$
\chi_{0}^{\left(q_{i}\right)}\left(\operatorname{Frob}_{p}\right)= \begin{cases}0 & \text { if } p \equiv 2(\bmod 3), \\ 0 & \text { if } p \equiv 1(\bmod 3) \text { and }\left(\frac{2}{p}\right)_{3} \neq 1, \\ 6 \quad & \text { if } p \equiv 1(\bmod 3),\left(\frac{2}{p}\right)_{3}=1, \\ & \left(\frac{q_{i}}{p}\right)_{3}=1, \\ -3 & \text { if } p \equiv 1(\bmod 3),\left(\frac{2}{p}\right)_{3}=1, \\ & \left(\frac{q_{i}}{p}\right)_{3} \neq 1 .\end{cases}
$$

Modulo all primes $p \equiv 1(\bmod 3)$ such that $p<$ $\left(\log N_{\chi_{0}^{\left(q_{i}\right)}}\right)^{1 / 2} \leq P_{i}$, the number $q_{i}$ was constructed to be a cubic residue. Further,

$$
\chi_{0}^{\left(q_{i}\right)}\left(\text { Frob }_{3}\right) 3^{-1}
$$

is of absolute value at most 2 . Thus,

$$
\log L\left(1, \chi_{0}^{\left(q_{i}\right)}\right)=6 \sum_{\substack{p \equiv 1(\bmod 3) \\\left(\frac{2}{p}\right)_{3}=1 \\ p<\left(\log N_{\chi_{0}^{\left(q_{i}\right)}}\right)^{1 / 2}}} \frac{1}{p}+O(1) .
$$

By the Čebotarev density theorem, the set of all primes such that $p \equiv 1(\bmod 3)$ and $\left(\frac{2}{p}\right)_{3}=1$ is of density $\frac{1}{6}$. We therefore have $\log L\left(1, \chi_{0}^{\left(q_{i}\right)}\right) \rightarrow \infty$ as soon as we may guarantee $N_{\chi_{0}^{\left(q_{i}\right)}} \rightarrow \infty$.

Since only a trivial character is missing, we have, by Corollary 4.6,

$$
\begin{aligned}
N_{\chi_{0}^{\left(q_{i}\right)}} & =N_{\left.\chi_{\operatorname{Pic}\left(S_{\bar{Q}}\left(q_{i}\right)\right.}\right)}=\left|\mathbf{D}\left(K_{1}\right) \mathbf{D}\left(K_{2}\right) \mathbf{D}\left(K_{3}\right) / 27\right|^{1 / 2} \\
& \geq\left|\mathbf{D}\left(K_{3}\right) / 27\right|^{1 / 2},
\end{aligned}
$$

where by choice of the coefficients,

$$
K_{3}=\mathbb{Q}\left(\zeta_{3}, \sqrt[3]{64 q_{i}}\right)=\mathbb{Q}\left(\zeta_{3}, \sqrt[3]{q_{i}}\right) .
$$

We have the estimate

$$
\begin{aligned}
\left|\mathbf{D}\left(K_{3}\right)\right|= & \left|\operatorname{Disc}\left(\mathbb{Q}\left(\zeta_{3}, \sqrt[3]{q_{i}}\right) / \mathbb{Q}\right)\right| \\
= & \operatorname{Disc}\left(\mathbb{Q}\left(\sqrt[3]{q_{i}}\right) / \mathbb{Q}\right)^{2} \\
& \times\left|N\left(\operatorname{Disc}\left(\mathbb{Q}\left(\zeta_{3}, \sqrt[3]{q_{i}}\right) / \mathbb{Q}\left(\sqrt[3]{q_{i}}\right)\right)\right)\right| \\
\geq & \operatorname{Disc}\left(\mathbb{Q}\left(\sqrt[3]{q_{i}}\right) / \mathbb{Q}\right)^{2} .
\end{aligned}
$$

According to [Marcus 77, Chapter 2, Exercise 41], we know that $\left|\operatorname{Disc}\left(\mathbb{Q}\left(\sqrt[3]{q_{i}}\right) / \mathbb{Q}\right)\right| \geq 3 q_{i}^{2}$.

Remark 8.2. Note that the estimate for $L\left(1, \chi_{0}^{\left(q_{i}\right)}\right)$ is the only point where we used the generalized Riemann hypothesis.

Observe in particular that we work with a version of Linnik's theorem that is true unconditionally. Here, the generalized Riemann hypothesis would lead to the much better exponent $2+\varepsilon$. However, this improvement is not necessary for our particular application.

\section{ACKNOWLEDGMENTS}

The computer part of this work was executed on the Sun Fire V20z Servers of the Gauss Laboratory for Scientific Computing at the Göttingen Mathematical Institute. Both authors are grateful to Prof. Y. Tschinkel for permission to use these machines as well as to the system administrators for their support.

\section{REFERENCES}

[Cassels 55] J. W. S. Cassels. "Bounds for the Least Solutions of Homogeneous Quadratic Equations." Proc. Cambridge Philos. Soc. 51 (1955), 262-264.

[Cohen 00] Henri Cohen. Advanced Topics in Computational Number Theory, Graduate Texts in Mathematics 193. New York: Springer, 2000.

[Colliot-Thélène and Sansuc 81] J.-L. Colliot-Thélène and J.-J. Sansuc. "On the Chow Groups of Certain Rational Surfaces: A sequel to a paper of S. Bloch." Duke Math. J. 48 (1981), 421-447.

[Colliot-Thélène et al. 87] J.-L. Colliot-Thélène, D. Kanevsky, and J.-J. Sansuc. "Arithmétique des surfaces cubiques diagonales." In Diophantine Approximation and Transcendence Theory (Bonn 1985), Lecture Notes in Mathematics 1290, pp. 1-108. Springer: Berlin, 1987. 
[Dokchitser 04] T. Dokchitser. "Computing Special Values of Motivic L-Functions." Experiment. Math. 13 (2004), $137-149$

[Duke 03] W, Duke. "Extreme Values of Artin L-Functions and Class Numbers." Compositio Math. 136 (2003), $103-115$.

[Elsenhans and Jahnel 06] A.-S. Elsenhans and J. Jahnel. "The Asymptotics of Points of Bounded Height on Diagonal Cubic and Quartic Threefolds." In Algorithmic Number Theory, Lecture Notes in Computer Science 4076, pp. 317332. Berlin: Springer, 2006.

[Elsenhans and Jahnel 07] A.-S. Elsenhans and J. Jahnel. "On the Smallest Point on a Diagonal Quartic Threefold." J. Ramanujan Math. Soc. 22 (2007), 189-204.

[Elsenhans and Jahnel 10a] A.-S. Elsenhans and J. Jahnel. "Experiments with General Cubic Surfaces." In Algebra, Arithmetic, and Geometry, Vol. 1: In Honor of Yu, edited by Y. Tschinkel and Y. Zarhin, pp. 637-654, Progress in Mathematics 269, Boston: Birkhaäuser, 2007.

[Elsenhans and Jahnel 10b] A.-S. Elsenhans and J. Jahnel. "Estimates for Tamagawa Numbers of Diagonal Cubic Surfaces." Journal of Number Theory (2010), doi:10.1016/j.jnt.2010.01.005.

[Heath-Brown 92] D. R. Heath-Brown. "Zero-Free Regions for Dirichlet $L$-Functions, and the Least Prime in an Arithmetic Progression." Proc. London Math. Soc. 64 (1992), 265-338.

[Ireland and Rosen 82] K. F. Ireland and M. I. Rosen. A Classical Introduction to Modern Number Theory, Graduate Texts in Mathematics 84. New York: Springer, 1982.
[Lavrik 67] A. F. Lavrik. "Functional Equations of the Dirichlet Functions" (Russian). Izv. Akad. Nauk SSSR, Ser. Mat. 31 (1967), 431-442.

[Manin 74] Yu. I. Manin. Cubic Forms. Amsterdam: NorthHolland, 1974.

[Marcus 77] D. A. Marcus. Number Fields. New York: Springer, 1977.

[Murty] M. R. Murty. "Applications of Symmetric Power L-Functions." In Lectures on Automorphic L-Functions, Fields Inst. Monogr. 20, pp. 203-283. Providence: Amer. Math. Soc., 2004.

[Neukirch 99] J. Neukirch. Algebraic Number Theory, Grundlehren der Mathematischen Wissenschaften 322. Berlin: Springer, 1999.

[Peyre 95] E. Peyre. "Hauteurs et mesures de Tamagawa sur les variétés de Fano." Duke Math. J. 79 (1995), 101-218.

[Peyre and Tschinkel 01] E. Peyre and Y. Tschinkel. "Tamagawa Numbers of Diagonal Cubic Surfaces, Numerical Evidence." Math. Comp. 70 (2001), 367-387.

[Press et al. 86] W. H. Press, B. P. Flannery, S. A. Teukolsky, and W. T. Vetterling. Numerical Recipes: The Art of Scientific Computing. Cambridge, UK: Cambridge University Press, 1986.

[Siegel 69] C. L. Siegel. "Abschätzung von Einheiten." Nachr. Akad. Wiss. Göttingen, Math.-Phys. Kl. II 1969 (1969), $71-86$.

[Stark 75] H. M. Stark. "Class Fields for Real Quadratic Fields and L-Series at 1." In Algebraic Number Fields: LFunctions and Galois Properties (Proc. Sympos., Durham, 1975), pp. 355-375. London: Academic Press, 1977.

Andreas-Stephan Elsenhans, Universität Bayreuth, Mathematisches Institut, Universitätsstraße 30, D-95447 Bayreuth, Germany, stephan.elsenhans@uni-bayreuth.de, http://www.staff.uni-bayreuth.de/ btm216.

Jörg Jahnel, Fachbereich 6, Mathematik, Universität Siegen, Walter-Flex-Straße 3, D-57068 Siegen, Germany, jahnel@mathematik.uni-siegen.de, http://www.uni-math.gwdg.de/jahnel.

Received November 19, 2008; accepted in revised form June 30, 2009. 